\title{
Clinical psychology and amyotrophic lateral sclerosis
}

\author{
Francesco Pagnini ${ }^{1}, 2 *$, Gabriella Rossi ${ }^{3}$, Christian Lunetta ${ }^{3}$, Paolo Banfi ${ }^{3}$ and Massimo Corbo ${ }^{3}$ \\ Department of Psychology, Catholic University of Milan, Milan, Italy \\ 2 Ph.D Program in Clinical Psychology, University of Bergamo, Bergamo, Italy \\ ${ }^{3}$ Neuromuscular Omnicentre, Fondazione Serena Onlus, Ospedale Niguarda Cà Granda, Milan, Italy
}

\section{Edited by:}

Edward Callus, Istituto di Ricovero e Cura a Carattere Scientifico, Policlinico San Donato, Italy

\section{Reviewed by:}

Lara Bellardita, Istituto di Ricovero e Cura a Carattere Scientifico Istituto

Nazionale dei Tumori, Italy; Università Cattolica del Sacro Cuore, Italy

Gianluca Castelnuovo, Istituto di

Ricovero e Cura a Carattere Scientifico

Istituto Auxologico Italiano, Italy;

Università Cattolica del Sacro Cuore,

Italy

Gian Mauro Manzoni, Istituto

Auxologico Italiano Istituto di Ricovero

e Cura a Carattere Scientifico, Italy

${ }^{*}$ Correspondence:

Francesco Pagnini, Dipartimento di

Psicologia (studio 99), Università

Cattolica del Sacro Cuore, Via Nirone,

1520123 Milan, Italy.

e-mail: francesco.pagnini@unicatt.it
Amyotrophic lateral sclerosis is a fatal and progressive disease, characterized by progressive muscles weakness, with consequent loss of physical capacities. Psychologists can play an important role in ALS care, by providing clinical activities in every step of the disease, including support and counseling activities directed to patients, their caregivers and to physicians.

Keywords: amyotrophic lateral sclerosis, clinical psychology, neurological illness, psychological support, burnout

\section{INTRODUCTION}

Amyotrophic lateral sclerosis (ALS) is a progressive and fatal neurodegenerative disease affecting motor neurons in the anterior horn of the spinal cord, the brainstem and the motor cortex with a pattern of progression not shared by other fatal diseases (Mitchell and Borasio, 2007). It is clinically characterized by progressive increasing weakness leading to death by respiratory insufficiency usually within three years (Haverkamp et al., 1995). ALS typically manifests itself as a muscle wasting or weakness in a limb or bulbar regions (Talbot, 2002). It then progresses to pervasive muscle weakness with a combination of upper and lower motor neuron dysfunction. Patients become relentlessly immobile, develop an impaired speech, often leading to social isolation. In the late stages of the disease, progressing paralysis can result in a "locked-in" state in which only residual muscular movement is possible (Borasio et al., 2001), but the intellect and the personality usually remain unimpaired.

The incidence of ALS is reported to be between 1.5 and 2.7 per 100,000 population/year (average 1.89 per 100,000/year) in Europe and North America, with a uniform incidence across these countries (Worms, 2001). The mean age of onset for ALS is between 55 to 65 years, with a $5 \%$ of cases that had an onset before the age of 30 years (Haverkamp et al., 1995). Although most cases of ALS are sporadic, about 5\% of cases have a family history of ALS (Mulder et al., 1986).

So far it is not possible to stop the progression of the disease therefore the primary goal in caring for patients with ALS is often considered to be the enhancement of their quality of life (Simmons, 2005).
Quality of life is generally defined in its biopsychosocial issues, which means an integration of physical and biological aspects (i.e., symptoms of illness or wellness), psychological aspects (i.e., emotions and thoughts), and social aspects (i.e., family relationships, social support...) (Engel, 1977).

All ALS patients show a similar clinical evolution, while loss of functions can have a different onset and has different times of worsening. Given the devastating nature of the disease, the access to a psychological support can be highly important. As well as for other very serious illness (i.e., cancer), psychological support should provide a scaffolding action, with a safe emotional space where people can express their feelings and emotions and be accompanied in the analysis of pain and fears. Psychologists can play an extremely active role in the rehabilitation process of ALS patients due to their capacity in providing a specific support for every phase in the disease progression.

Furthermore, psychologists should help not only patients and families in understanding what is happening, but they can also play a key role in supporting the other members of the multiprofessional team in their endeavor managing such an over complex framework.

\section{DIAGNOSTIC COMMUNICATION}

The way in which ALS diagnosis is communicated to the patients appears to be of the most importance in determining their initial reaction (Simmons, 2005). Communication of ALS diagnosis may cause major repeated psychological trauma which the patient will 
have to cope with, using defenses and adaptation mechanisms. Clinical management of ALS should include knowledge, understanding, and acceptance of these mechanisms. This could be challenging for physicians, who may be unprepared to break bad news (Buckman, 1996), mostly because of a lack of emotional management training (Pagnini et al., 2009). Guidelines for breaking the news to a patient with ALS (Borasio et al., 1998) also suggest the proposal of a psychological support.

The ALS diagnosis is a devastating label. Right after the communication of the diagnosis, patients often need a "room" in which they can express their emotions, usually despair, regarding what they will have to inevitably face in the future.

As mentioned above, the diagnostic communication of the illness is a crucial and emotionally relevant moment for the patient. The presence of a psychologist in the medical staff can allow the patients to express their feelings and fears in a better way compared to what they would have done with the only presence of a physician. A patient-centered service should be very careful in diagnostic communication and the clinical team should work together to enhance the quality of the patient-clinician relationship, with a particular focus on the diagnostic and prognostic communication processes.

Relatives and close caregivers of ALS patients should be able to receive psychological support from the very first period of disease. The label "ALS" is a huge burden to be carried, both by patients and their relatives. In the beginning they need to cope with bereavement, caused by the knowledge about the progressive loss of motor functions and the awareness that, within a few years, the patient will probably pass away.

Psychological support for families should be both expressive (promoting mourning management) and practical, helping relatives with discussion and practical suggestions. For example, people can be worried and confused about "if" and "how" to communicate information regarding the disease to their small children and this could be object of practical analysis in psychological counseling.

\section{DISEASE PROGRESSION}

During illness progression, the awareness of an emotionally safe "room", offered by the psychologist, is a great resource for patients and caregivers, improving their quality of life. During counseling they can explore their needs, feel free to voice their emotions, guaranteeing a cathartic effect. People can be seen alone, with their partner or as a family. At the same time it is also possible to manage other practical troubles that cause personal, familiar, or social diseases, problems that could be unrelated to the apparition of ALS symptoms. Sometimes clinicians need to face pre-existent issues, for example couple problems, that interact with the new medical condition.

Spirituality and existential well-being issues may be addressed and explored in these interviews, with respect and the awareness that they are very powerful resources in the coping with illness and the shadow of death. It is sometimes useful for psychologists to send patients to a spiritual counselor, according to the patients' beliefs (i.e., a priest, a monk, maybe a philosopher...).

The importance of spiritual care is usually underestimated (Lambert, 2006). Spiritual care should encompass the whole family as a means of preventing complicated bereavement.
Throughout the course of the disease, a balance should be maintained by physicians between anticipating onsets of loss of functions and introducing life support measures, on the one hand, and respecting the patient's psychological state, life plans, and environment, on the other.

As far as patient worsens, he loses physical functions. Medical knowledge is unable to reverse this process, but it can offer auxiliaries to compensate, like walkers, manual wheelchairs, or power chairs. Many people look at these auxiliaries as a "painful goodbye" to their autonomy, a limitation of freedom. In a clinical relationship these feelings can be managed and elaborated.

Furthermore, ALS patients will, sooner or later, face the loss of speech and voice. Usually there is an initial decrease of speech volume, followed by a change in the voice, becoming more confused. This may be followed by complete loss of speech. In order to prevent devastating psychological and social consequences, patients and caregivers should access soon to other forms of communication. In addition to technological solutions, like computerized communicators, there are different ways to communicate, like hand signals, head nodding, sign language, etc. (Simmons, 2005). The psychologist should facilitate the awareness that living without speech is not living without communicating. Furthermore, it is essential that caregiver-patient couple find a way to easily interact between them and this could be a task for psychologists.

\section{VENTILATORY AND NUTRITIONAL MANAGEMENT}

At a certain point of the illness, ALS patients' respiratory capacity reduces. The ventilatory ability gets worst, providing a minor amount of oxygen. Respiratory support is usually provided by noninvasive ventilation (NIV) or invasive ventilation via tracheotomy. NIV is the provision of ventilatory support through the patient's upper airway using a mask or similar device, while tracheotomy is a surgical procedure on the neck to open a direct airway through an incision in the trachea. NIV is usually initially used for sporadic nocturnal support to alleviate symptoms of nocturnal hypoventilation. As respiratory function worsens, patients tend to require increasing daytime NIV support and eventually continuous support.

Together with the emotional strain due to the decrease of respiratory functions, many patients report a rejection of the mask, describing an experience similar to claustrophobia, where they feel suffocating, feeling an increasing anxiety, and anguish. The psychologist can allow a controlled expression of these feelings, reducing anxious symptomatology. Respiratory anxiety can be treated with relaxation techniques, in particular with those related to breathe listening and control.

Bulbar dysfunctions lead to risk for malnutrition and dehydration. Nutrition troubles in ALS usually begin with liquids and then progresses involving other consistencies. Patient's capacity to swallow food decreases as tongue weaknesses, while coughing and choking increase. Initially management of this dysphagia consists in urging a change in food consistency, but when this method is not enough, it is proposed a feeding gastrostomy tube to the patient (PEG or RIG).

\section{IMPORTANT CHOICES}

Despite a variety in disease progression, sooner or later all ALS patients must face important decisions. In particular, choices about nutrition management or a tracheostomy to keep breathing. 
These choices are highly important and hard to be taken, since they will have an important impact on the patients' lives. Patients should be able to make a choice with the consciousness of medical issues, communicated by physicians, but they also need time to express their fears and doubts. When patient have to decide about an intervention, an informed consent must be signed. Banfi et al. (2009) propose a two-steps informed consent, with the communication of clinically relevant information made by physician and a second phase, after some hours or some days, where patients can explore feelings and fears to the psychologist. Only in this second step the consent will be signed.

\section{END-OF-LIFE PHASE}

Psychological support becomes even more important in the end-oflife phase of the disease. Psychologists need to be aware of potential difficulties in the bereavement outcome for surviving relatives and/ or carers. Difficult questions are often asked to clinicians from both patients and caregivers, questions typically related to the suffering management at the end of life. In this period the emotional strain is very high and can promote the breakdown of a psychological equilibrium in pre-morbid subjects. There could be requests for assistance to die as a means of stopping the suffering. Answers to these questions should be given sensitively and honestly, considering the ability of the individuals to understand and manage the information that they are asking for.

After the patient's death continuous counseling is often needed in order to cope with the grief of mourning.

The death of a person has a lasting effect on his family and not all people are able to cope well with the situation (Martin and Turnbull, 2001). Grief and bereavement support is integral to ALS care, but a study conducted in the US indicates that the support provided by ALS centers is often inadequate (Hebert et al., 2005). Measures such as the possibility to talk to the psychologist or a letter of condolence can improve this support. A letter of condolence can help a bereaved family and tie-up the relationship between the physician and the late patient's family (Bedell et al., 2001).

\section{CAREGIVERS}

The burden experienced by ALS caregivers is severe, as the one experienced in caring for any other neurological disorder. Hecht et al. (2003) analyzed the burden of ALS carers, comparing it with the one experienced by carers of other diseases. They found a lower level of care burden for ALS, compared with dementia or mixed neuropsychiatric and internal diseases, but it was correlated with functional impairment. Personal and social restrictions and

\section{REFERENCES}

Banfi, P., Rossi, G., Pagnini, F., Cellotto, N., Gorni, K. O., Lunetta, C., Roma, E., Castelnuovo, G., Molinari, E., and Corbo, M. (2009). Towards a multistep informed consent: considerations and proposals for a good practice. Clin. Ter. 160, 425-426.

Bedell, S. E., Cadenhead, K., and Graboys, T. B. (2001). The doctor's letter of condolence. N. Engl. J. Med. 344, 1162-1164.

physical and emotional problems were the main burden components. Given that the burden of care increases with the functional impairment, these researchers suggest that support for caregivers has to start sooner.

Caregivers spend a lot of time for day caring, sometimes more than $11 \mathrm{~h}$ a day, despite having other assistance in the home (Krivickas et al., 1997) and those who believe they cannot leave the patient are the most distressed (Rabkin et al., 2005). ALS caregivers note many changes in patients, including increased tension, less frequent vacations, decreased time for themselves, increased anxiety, and decreased time for recreational activities (Mitsumoto, 2002).

Together with emotional strain, care in ALS requires a certain amount of physical effort, in particular during the advanced phases of the disease. Patients need assistance to make movements and to get up and providing this could be a problem for people with physical impairments. This is particularly true for old caregivers, with some age-related troubles.

The progressive nature of the disease promote dependence of the patient upon a primary caregiver and it is important to identify factors relevant to quality of life for both patient and caregiver (Lo Coco et al., 2005) and to improve these as much as possible. There is some evidence supporting the idea that caregivers influence the mental and physical status of patients with ALS (Chio et al., 2004). The therapeutic team should be aware of the strengths and deficiencies of the caregivers and should help them in improving their coping skills. Since concordance of depression and distress levels between patients and caregivers is high, attention to the mental health of the caregiver could alleviate the patient's distress as well (Rabkin et al., 2000).

Perceived social support is an important predictor of carer distress (Goldstein et al., 2006), marital relationship satisfaction (O'Connor et al., 2008), and patient's quality of life (Chio et al., 2004). However, the maintain of a network of social support may be difficult to accomplish, as friends often stop visiting or became awkward around the patient as symptoms increase in severity (Cobb and Hamera, 1986). Even if the enhancement of relationships may be considered as a task for other specialists, clinicians could work with caregivers and patients in order to improve their social networks, when this could have a positive influence on their well-being.

\section{ACKNOWLEDGMENTS}

The MAPPA DM SLA Project (Modelli di Assistenza (ordinario ed intensivo) Per Persone Affette da Distrofia Muscolare e Sclerosi Laterale Amiotrofica) was supported by the "Fondazione Cariplo" Italian private foundation.

and Mutani, R. (2004). A cross sectional study on determinants of quality of life in ALS. J. Neurol. Neurosurg. Psychiatr. 75, 1597-1601.

Cobb, A. K., and Hamera, E. (1986). Illness experience in a chronic disease-ALS. Soc. Sci. Med. 23, 641-650.

Engel, G. L. (1977). The need for a new medical model: a challenge for biomedicine. Science 196, 129-136.

Goldstein, L. H., Atkins, L., Landau, S., Brown, R., and Leigh, P. N. (2006).
Predictors of psychological distress in carers of people with amyotrophic lateral sclerosis: a longitudinal study. Psychol. Med. 36, 865-875.

Haverkamp, L. J., Appel, V., and Appel, S. H. (1995). Natural history of amyotrophic lateral sclerosis in a database population. Validation of a scoring system and a model for survival prediction. Brain 118(Pt 3), 707-719.

Hebert, R. S., Lacomis, D., Easter, C., Frick, V., and Shear, M. K. (2005). 
Grief support for informal caregivers of patients with ALS: a national survey. Neurology 64, 137-138.

Hecht, M. J., Graesel, E., Tigges, S., Hillemacher, T., Winterholler, M., Hilz, M. J., Heuss, D., and Neundorfer, B. (2003). Burden of care in amyotrophic lateral sclerosis. Palliat. Med. 17, 327-333.

Krivickas,L.S.,Shockley,L., and Mitsumoto, H. (1997). Home care of patients with amyotrophic lateral sclerosis (ALS). J. Neurol. Sci. 152(Suppl. 1), S82-S89.

Lambert, R. (2006). "Spiritual care," in Palliative Care in Amyotrophic Lateral Sclerosis: From Diagnosis to Bereavement, 2nd Edn. eds. D. Oliver et al. (Oxford: Oxford University Press), 169-185.

Lo Coco, G., Lo Coco, D., Cicero, V., Oliveri, A., Lo Verso, G., Piccoli, F., and La Bella, V. (2005). Individual and health-related quality of life assessment in amyotrophic lateral sclerosis patients and their caregivers. J. Neurol. Sci. 238, 11-17.
Martin, J., and Turnbull, J. (2001). Lasting impact in families after death from ALS. Amyotroph. Lateral Scler. Other Motor Neuron Disord. 2, 181-187.

Mitchell, J. D., and Borasio, G. D. (2007). Amyotrophic lateral sclerosis. Lancet 369, 2031-2041.

Mitsumoto, H. (2002). Caregiver assessment: summary. Amyotroph. Lateral Scler. Other Motor Neuron Disord. 3(Suppl. 1), S31-S34.

Mulder, D. W., Kurland, L. T., Offord, K. P., and Beard, C. M. (1986). Familial adult motor neuron disease: amyotrophic lateral sclerosis. Neurology 36, 511-517.

O'Connor E, J., McCabe, M. P., and Firth, L. (2008). The impact of neurological illness on marital relationships. J. Sex Marital Ther. 34, 115-132.

Pagnini,F.,Manzoni,G.M., and Castelnuovo, G. (2009). Emotional intelligence training and evaluation in physicians. JAMA 301, 600; author reply 601 .
Rabkin, J. G., Albert, S. M., Del Bene, M. L., O'Sullivan, I., Tider, T., Rowland, L. P., and Mitsumoto, H. (2005). Prevalence of depressive disorders and change over time in late-stage ALS. Neurology 65, 62-67.

Rabkin, J. G., Wagner, G. J., and Del Bene, M. (2000). Resilience and distress among amyotrophic lateral sclerosis patients and caregivers. Psychosom. Med. 62, 271-279.

Simmons, Z. (2005). Management strategies for patients with amyotrophic lateral sclerosis from diagnosis through death. Neurologist 11, 257-270.

Talbot, K. (2002). Motor neurone disease. Postgrad. Med. J. 78, 513-519.

Worms, P. M. (2001). The epidemiology of motor neuron diseases: a review of recent studies. J. Neurol. Sci. 191, 3-9.

Conflict of Interest Statement: The authors declare that the research was conducted in the absence of any commercial or financial relationships that could be construed as a potential conflict of interest.

Received: 26 April 2010; paper pending published: 14 May 2010; accepted: 30 June 2010; published online: 21 July 2010.

Citation: Pagnini F, Rossi G, Lunetta C, Banfi $P$ and Corbo M (2010) Clinical psychology and amyotrophic lateral sclerosis. Front. Psychology 1:33. doi: 10.3389/ fpsyg.2010.00033

This article was submitted to Frontiers in Psychology for Clinical Settings, a specialty of Frontiers in Psychology.

Copyright (C) 2010 Pagnini, Rossi, Lunetta, Banfi and Corbo. This is an open-access article subject to an exclusive license agreement between the authors and the Frontiers Research Foundation, which permits unrestricted use, distribution, and reproduction in any medium, provided the original authors and source are credited. 